\title{
The optical properties of the cobalt nanoparticles in the transparent condensed matrices
}

\author{
M. V. Ananyeva ${ }^{1}$, A. V. Kalenskii ${ }^{1}$, A. A. Zvekov², A. P. Nikitin ${ }^{2}$, I. Yu. Zykov ${ }^{1}$ \\ ${ }^{1}$ Kemerovo state University, Kemerovo, 650043, Russia, \\ ${ }^{2}$ Institute of Coal Chemistry and Material Science SB RAS, Kemerovo, 650000, Russia
}

kriger@kemsu.ru

\author{
PACS 61.46.+w, 82.33.Vx
}

DOI 10.17586/2220-8054-2015-6-5-628-636

\begin{abstract}
The absorption and scattering efficiency factors for cobalt nanoparticles over wavelengths ranging from 400 $1200 \mathrm{~nm}$ were calculated. The maximum values and corresponding radii of the absorption efficiency were shown to be dependent upon the incident light wavelength. The highest scattering factor values in the studied spectral range were between 2.2 and 2.4. If the absorptivity of the matrix increases, absorption of the light by cobalt nanoparticles begins to dominate over scattering process. The obtained results allow us to predict the optical properties for composite materials based on a transparent matrix with cobalt nanoparticles, which is essential for the accurate modeling of such systems' behavior under laser irradiation.
\end{abstract}

Keywords: optical properties, Mie theory, nanoparticles, cobalt, composite materials, laser irradiation.

Received: 5 July 2015

Revised: 27 September 2015

\section{Introduction}

Nanoparticles have unique thermochemical, physical and optical properties. Their inclusion into condensed matrices significantly changes the properties of the obtained composite materials. The composites, based on metal nanoparticles and transparent matrix, are of the great interest for the optical physics and optoelectronics [1]. Both experimental and theoretical investigations of such composites' optical properties are presented in [2-4]. The problem is important today because of the possible practical use of the absorption and scattering of light by nanoparticles in timing devices of nonlinear optical equipment [5], application to the thermal cancer therapy, and optical detonators $[6,7]$.

It is known that iron-group element nanoparticles may be used in the high-speed optical devices $[7,8]$. Aluminium nanoparticles in pentaerythritol tetranitrate (PETN) reduce the explosive decomposition threshold by more than 100-fold in case of initiation by the first and second harmonics of the Nd:YAG laser $[6,9,10]$. The possibility of using cobalt nanoparticle-based composites in secondary explosives as an optical detonator cup was mentioned in $[3,11,12]$. Methods for synthesizing cobalt nanoparticles with definite sizes have been previously described [13]. In order to calculate the illumination intensity in the composite's volume, it is necessary to take into account multiple scattering of light by the metal nanoparticles $[14,15]$. This point was verified experimentally for the pressed PETN-aluminium pellets [4]. In order to find the optimal material for an optical detonator cup and compositions for the nonlinear optical equipment, it is necessary to calculate the relationships of the absorption and scattering efficiency factors by varying particle size and the wavelength of the incident light [16]. 


\section{Calculation procedure and Results}

The interaction of metal nanoparticles and electromagnetic radiation is traditionally described by using the absorption efficiency $\left(Q_{\text {abs }}\right)$ and scattering efficiency factors $\left(Q_{\text {sca }}\right)$. These dimensionless parameters are equal to the ratio of the particle's cross-section and its geometrical cross-section $Q=\sigma / \sigma_{g}$. The $Q_{\text {abs }}$ was calculated in terms of Mie theory as the difference of the absorption efficiency $\left(Q_{\text {ext }}\right)$ and scattering efficiency factors $\left(Q_{\text {sca }}\right)$ [16-18].

The main parameters of the theory are the complex refractive index $\left(m_{i}\right)$ of cobalt, which depends on the wavelength of the incident light $(\lambda)$, and the refractive index of the medium $\left(m_{0}\right)$ [19]. These data over the range $400-1210 \mathrm{~nm}$ with increments of $\sim 100 \mathrm{~nm}$ are presented in [20]. In order to calculate the absorption and scattering efficiency factors for the wavelengths, which were not presented in [19], work we used the spline interpolation with $1 \mathrm{~nm}$ increments, obtaining a smooth dependence $m_{i}(\lambda)$ in this manner $[18,19]$. The range of nanoparticle radii studied is dictated by their potential use in optic initiation systems where values of $20<R<200 \mathrm{~nm}$ are utilized.

Figures 1 and 2 show the dependences of the absorption efficiency $\left(Q_{\mathrm{abs}}\right)$ and scattering efficiency factors $\left(Q_{\text {sca }}\right)$ respectively on the cobalt particle's radius in a transparent medium with $m_{0}$ equal to 1.54 calculated for the light wavelengths of 400, 600, 800, 1000, and $1200 \mathrm{~nm}$.

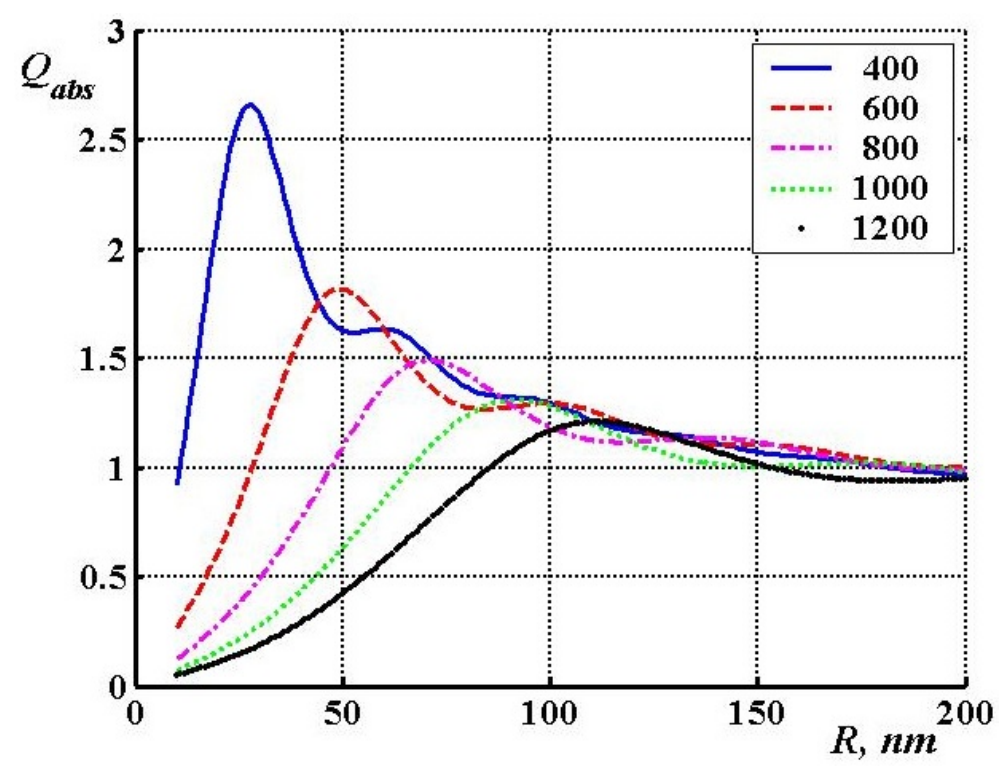

FIG. 1. The dependence of the absorption efficiency factor of the cobalt nanoparticles in the PETN matrix on their radius for the wavelengths in $\mathrm{nm}$ presented in the legend.

This refractive index was chosen as a typical value for dielectric media and is equal to the refractive index of pentaerythritol tetranitrate (PETN), which is one of the most important compounds among secondary explosives. As seen, $Q_{\mathrm{abs}}$ values increase for small particle radii values and tend to steady-state value with oscillations in the $R \rightarrow \infty$ limit [21,22]. The dependence has a well-marked maximum which tends to shift to larger radii when the wavelength increases. At the same time, the maximum's amplitude decreases significantly. The dependence $Q_{\text {sca }}$ vs. $R$ increases for the small radii, then it achieves constant value and remains close to that value with minor fluctuations. The curve $Q_{\text {sca }}(R)$ has several local maxima. One can see the shift of the principal maximum position in Fig. 3. Enumerating the maxima from 


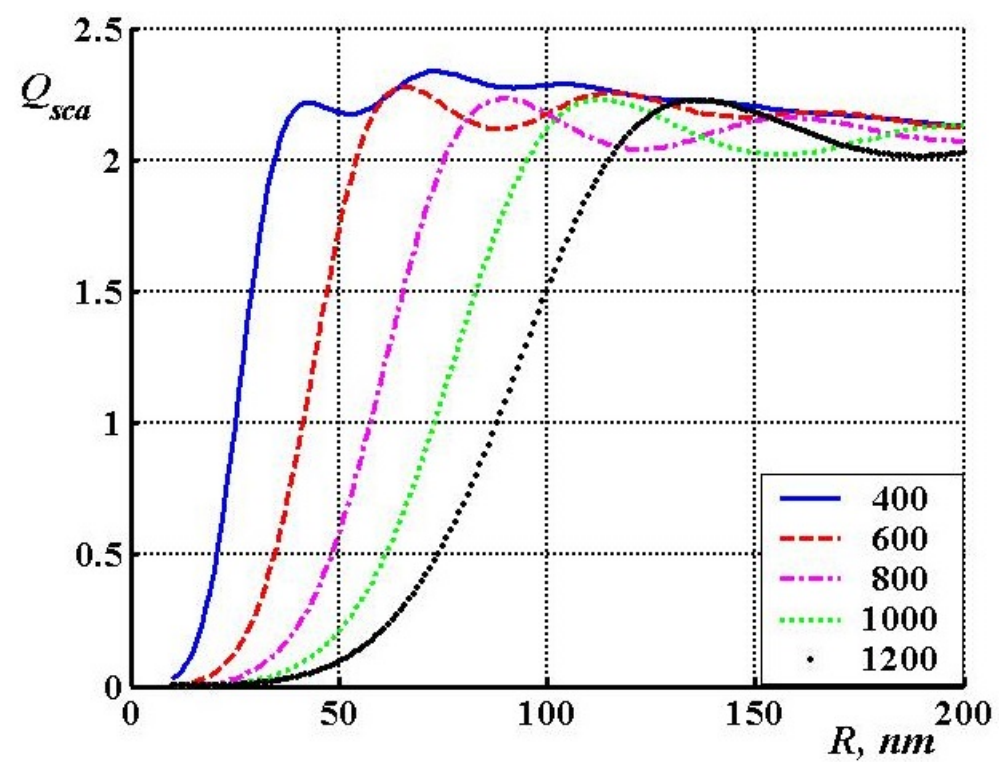

FIG. 2. The dependence of the scattering efficiency factor of the cobalt nanoparticles in the PETN matrix on their radius for the wavelengths in $\mathrm{nm}$ presented in the legend.

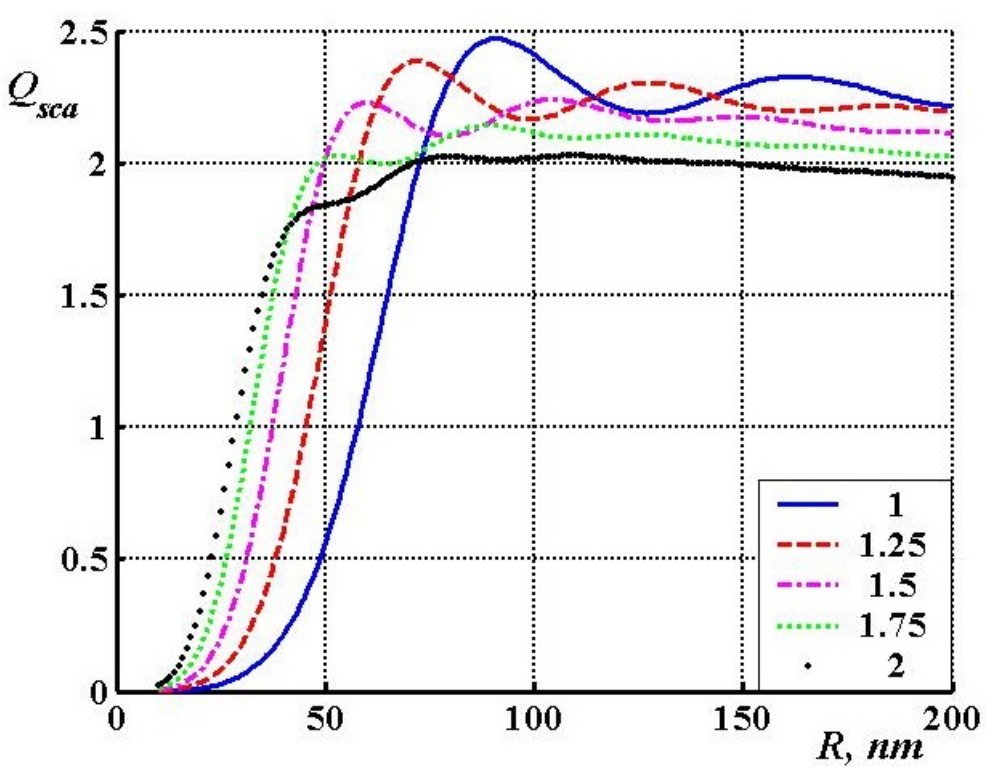

FIG. 3. The dependence of the scattering efficiency factor of the cobalt nanoparticles for of the second harmonics of Nd:YAG laser irradiation. The refractive indexes of the media are $1,1.25,1.5,1.75$, and 2

small to higher $R$ values it is seen that for $\lambda>463 \mathrm{~nm}$, the principal maximum is the first one, while in the spectral range $\lambda \leq 463 \mathrm{~nm}$ the highest maximum is the second one.

Figures 3 and 4 show the dependences of the absorption and scattering efficiency factors of the cobalt nanoparticles upon their radii for $532 \mathrm{~nm}$ light and for different refractive indices. This value of $\lambda$ coincides with the second harmonics wavelength for the Nd:YAG laser, which is widely used in many applications [6-8]. The displays show that increasing the medium's refractive index results in an increase of the maximum amplitude for $Q_{\mathrm{abs}}(R)$ and a shift to smaller radii values. The increasing of the refraction index of the media results in the shift 


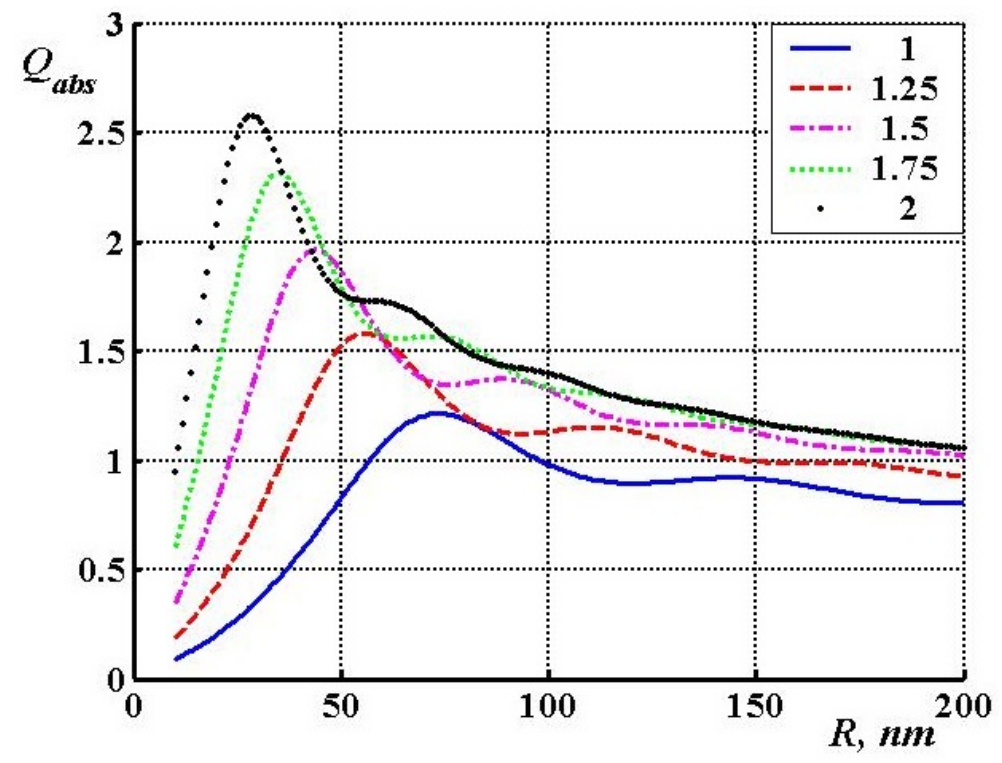

FIG. 4. The dependence of the cobalt nanoparticle absorption factor in case of irradiation of the second harmonic of Nd:YAG laser, refractive indexes of the media are $1,1.25,1.5,1.75$, and 2

of the initial ascending part of the $Q_{\text {sca }}(R)$ curve to the smaller radii values. Additionally, the oscillations' amplitude decreases for larger particle radius values and one can see a small decrease for the $Q_{\text {sca }}$ stationary value when $R \rightarrow \infty$.

Based on the data presented above, the maximum values of absorption $\left(Q_{\text {abs max }}\right)$ and scattering efficiency factors $\left(Q_{\text {sca max }}\right)$ were calculated for cobalt nanoparticles for different wavelengths. These calculations for the positions of the maxima were done assuming a refractive index 1.54 for the medium. The results are presented in Table 1. These data show that the absorption exceeds the scattering for shorter wavelength values. The maximum values of absorption and scattering efficiency factor coincide for cobalt nanoparticles at a wavelength of $482.5 \mathrm{~nm}$. Increasing of wavelength from 400 up to $1200 \mathrm{~nm}$ results in monotonic $Q_{\text {abs max }}(\lambda)$ decreasing in $\sim 2.2$ fold, from 2.6589 to 1.2065 . If the wavelength increases, the radius of the particles with the largest absorption efficiency factor $\left(R_{\text {abs max }}\right)$ increases linearly from $27.7 \mathrm{~nm}$ for $\lambda=400 \mathrm{~nm}$ up to $111.5 \mathrm{~nm}$ for $\lambda=1200 \mathrm{~nm}$. For $Q_{\text {sca } \max }(\lambda)$, this dependence is dissimilar. $Q_{\text {sca max }}$ is almost independent of the wavelength and shows small oscillations in the vicinity of the stationary value. For all wavelengths, the values of $Q_{\text {sca max }}(\lambda)$ are $2.1992-2.3371$, and the minimum is observed at $\lambda=471 \mathrm{~nm}$. The radius of the nanoparticles having the greatest scattering efficiency factor for a given wavelength $\left(R_{\text {sca max }}\right)$ increases from $73.3 \mathrm{~nm}$ to $105.3 \mathrm{~nm}$ in the $400<\lambda<550 \mathrm{~nm}$ range and from $63.2 \mathrm{~nm}$ to $136.3 \mathrm{~nm}$ in the $575<\lambda<1200 \mathrm{~nm}$ range. In the $550-575 \mathrm{~nm}$ wavelength range, a two fold decreasing of $R_{\text {sca max }}$ values was observed. This might be explained by the fact that in the $400<\lambda<550 \mathrm{~nm}$ range, the second maximum dominates for the $Q_{\mathrm{sca}}(R)$ dependence, and if the wavelength is longer than $550 \mathrm{~nm}$, the first one dominates.

The contributions of the harmonics to the scattering efficiency factor were calculated for a wavelength of $532 \mathrm{~nm}$ and $m_{0}=1.54$ in an attempt to explain the shift of the maximum (Fig. 5). Letters $c$ denotes the electric-type oscillations of the electron density on the surface of the metal, letters $b$ - magnetic-type oscillations. The figures show the harmonics index: $1-$ dipole, 2 - quadrupole, 3 - octopole, etc. One can readily see that when the radius of the cobalt nanoparticles is less than $70 \mathrm{~nm}$, the first electric oscillation predominates. When the radius 
TABLE 1. Spectral regularities for maximum absorption and scattering factor values for cobalt nanoparticles

\begin{tabular}{|c|c|c|c|c|c|}
\hline$\lambda, \mathrm{nm}$ & $m_{i}$ & $Q_{\text {abs max }}$ & $R_{\text {abs max }}, \mathrm{nm}$ & $Q_{\text {sca max }}$ & $R_{\text {sca max }}, \mathrm{nm}$ \\
\hline 400 & $1.2000-3.0000 i$ & 2.6589 & 27.7 & 2.3371 & 73.3 \\
\hline 450 & $1.5070-3.1721 i$ & 2.3400 & 33.5 & 2.2070 & 85.0 \\
\hline 500 & $1.7000-3.4000 i$ & 2.1355 & 38.9 & 2.2079 & 95.3 \\
\hline 532 & $1.7932-3.5609 i$ & 2.0249 & 42.2 & 2.2286 & 101.7 \\
\hline 550 & $1.8430-3.6529 i$ & 1.9662 & 44.1 & 2.2402 & 105.3 \\
\hline 600 & $2.0000-3.9000 i$ & 1.8172 & 49.4 & 2.2777 & 66.0 \\
\hline 650 & $2.2148-4.1164 i$ & 1.6973 & 54.9 & 2.2787 & 71.8 \\
\hline 700 & $2.4500-4.3000 i$ & 1.6086 & 60.3 & 2.2613 & 77.8 \\
\hline 750 & $2.6628-4.4567 i$ & 1.5434 & 65.6 & 2.2469 & 83.7 \\
\hline 800 & $2.8700-4.6000 i$ & 1.4907 & 70.9 & 2.2333 & 89.6 \\
\hline 850 & $3.0914-4.7444 i$ & 1.4432 & 76.2 & 2.2196 & 95.6 \\
\hline 900 & $3.3000-4.9000 i$ & 1.3978 & 81.4 & 2.2134 & 101.4 \\
\hline 950 & $3.4667-5.0719 i$ & 1.3528 & 86.5 & 2.2191 & 107.2 \\
\hline 1000 & $3.6000-5.2500 i$ & 1.3096 & 91.6 & 2.2304 & 112.9 \\
\hline 1050 & $3.7178-5.4203 i$ & 1.2708 & 96.5 & 2.2411 & 118.7 \\
\hline 1064 & $3.7505-5.4647 i$ & 1.2611 & 97.9 & 2.2433 & 120.3 \\
\hline 1100 & $3.8381-5.5688 i$ & 1.2391 & 101.5 & 2.2463 & 124.5 \\
\hline 1150 & $3.9789-5.6816 i$ & 1.2170 & 106.5 & 2.2421 & 130.4 \\
\hline 1200 & $4.1580-5.7446 i$ & 1.2065 & 111.5 & 2.2255 & 136.3 \\
\hline & & & & & \\
\hline
\end{tabular}

increases, the contribution of the second electric and the first magnetic oscillations become noticeable, these oscillations become dominant when $R>100 \mathrm{~nm}$. Competition between the electric and magnetic oscillations results in two different effects:

i) the maximum on the dependence $Q_{\mathrm{abs}}(R)$ shifts with increasing wavelength;

ii) local maxima for the dependence $Q_{\text {sca }}(R)$ have almost the same amplitude and for large cobalt nanoparticle radii; there is almost no dependence on $R$. Since there are several oscillations of comparable intensity, their sum changes only slightly.

Figures 6 and 7 present spectral dependencies of the absorption and scattering efficiency factors for cobalt nanoparticles of different radii in a PETN matrix. The line descriptions and radii values are represented on the legend. For $30 \mathrm{~nm}$ nanoparticles, $Q_{\mathrm{abs}}$ decreases monotonically. For the $50 \mathrm{~nm}$ nanoparticles, a maximum for $Q_{\mathrm{abs}}$ occurs at $553 \mathrm{~nm}$ and that of the scattering efficiency factor at $400 \mathrm{~nm}$. Increasing the nanoparticle radius causes the appearance of a second maximum. It is worth pointing out the difference between our results and those obtained for gold nanoparticles [16]. Spectral regularities of the latter have a narrow band (half-width $\sim$ tens $\mathrm{nm}$ ) due to plasmon resonance. In the case of cobalt, there are wide bands in the absorption spectra and scattering efficiency factor, as for other iron-group elements $[7,8,15]$.

In the visible region of the spectrum and for larger cobalt nanoparticles, the standard deviation of the absorption and scattering factors is about $10-20 \%$. The weak dependence 


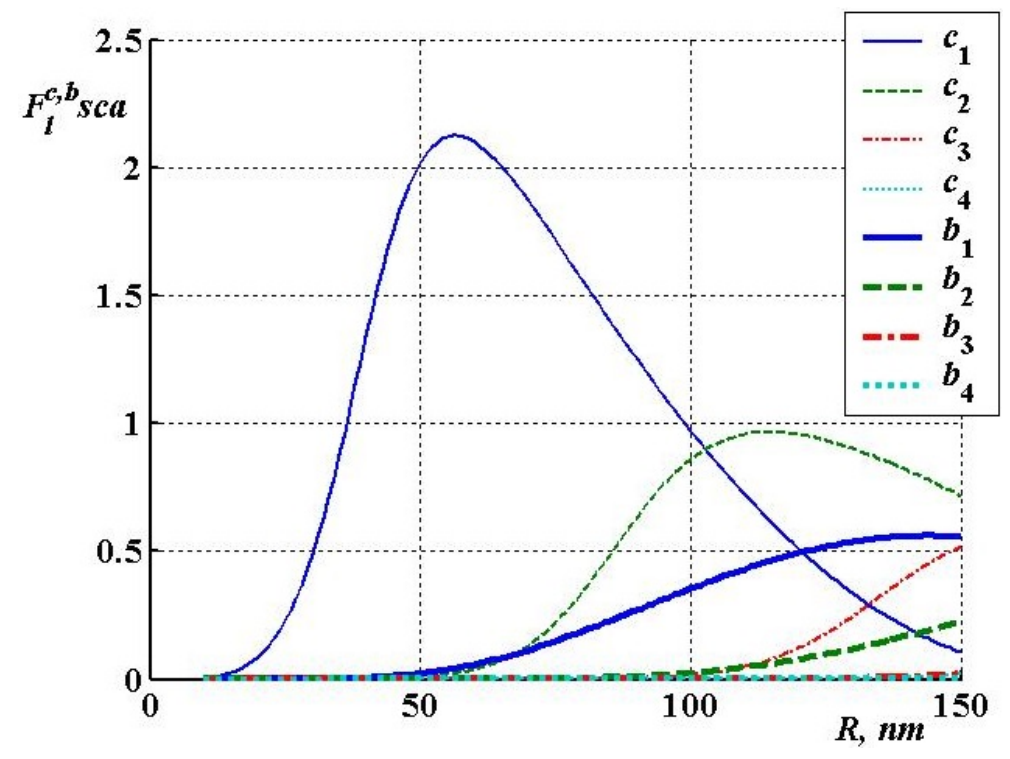

FIG. 5. Dependences of electric (c) and magnetic (b) oscillations of the scattering factor upon the cobalt nanoparticle radius at $532 \mathrm{~nm}$

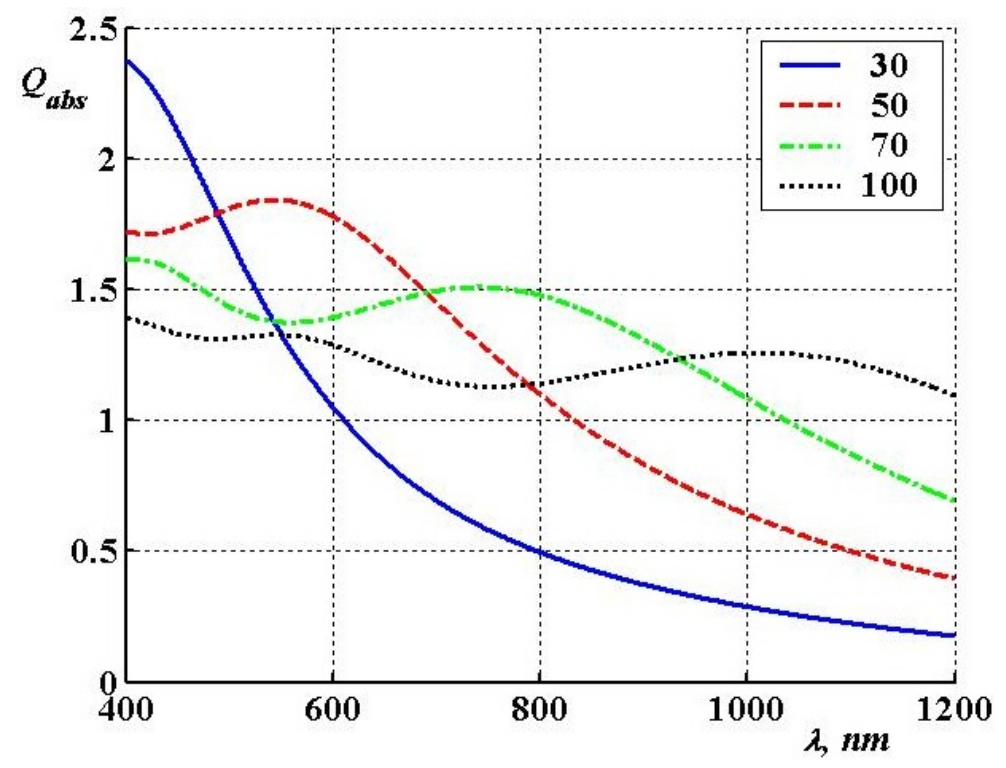

FIG. 6. Spectral dependencies of the absorption efficiency factors for cobalt nanoparticles with $30,50,70$, and $100 \mathrm{~nm}$ radii

of the mentioned parameters upon the wavelength proves that the composite materials, based on the transparent media and cobalt nanoparticles with radius of $\sim 100 \mathrm{~nm}$, are neutral-density filters. So, this differs from the gold or silver colloidal solutions, which are colored and are used as a band-pass filter. Thus, it appears that a light filter with cobalt nanoparticles decreases the light's intensity primarily by scattering but not because of the absorption.

Figure 8 displays the calculated spectral regularities for the absorptivity of $1.5 \mathrm{~nm}$ cobalt nanoparticles and the experimental values of the molar absorptivity $\varepsilon$ presented in [23]. The figure shows that the absorption predominates considerably over the scattering and the comparison of the extinction and the absorption is incorrect. As it follows from Fig. 8, in both cases, decreasing the wavelength results in increased absorptive properties. The region of 


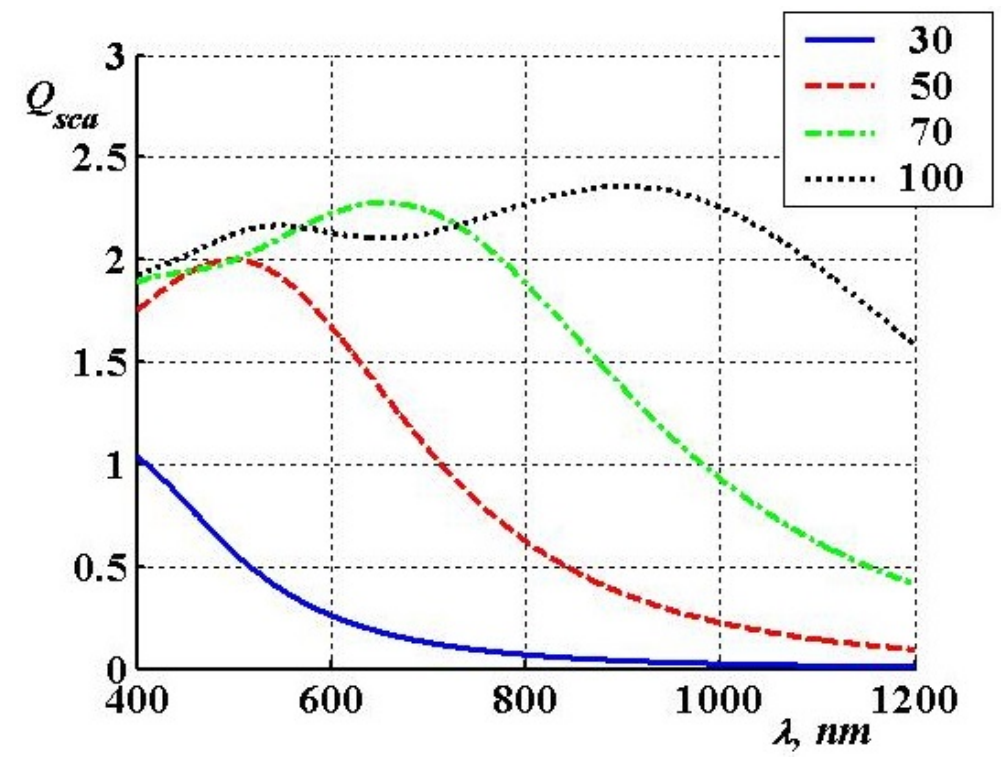

FIG. 7. Spectral dependencies of the scattering efficiency factors for cobalt nanoparticles with radii of $30,50,70$, and $100 \mathrm{~nm}$

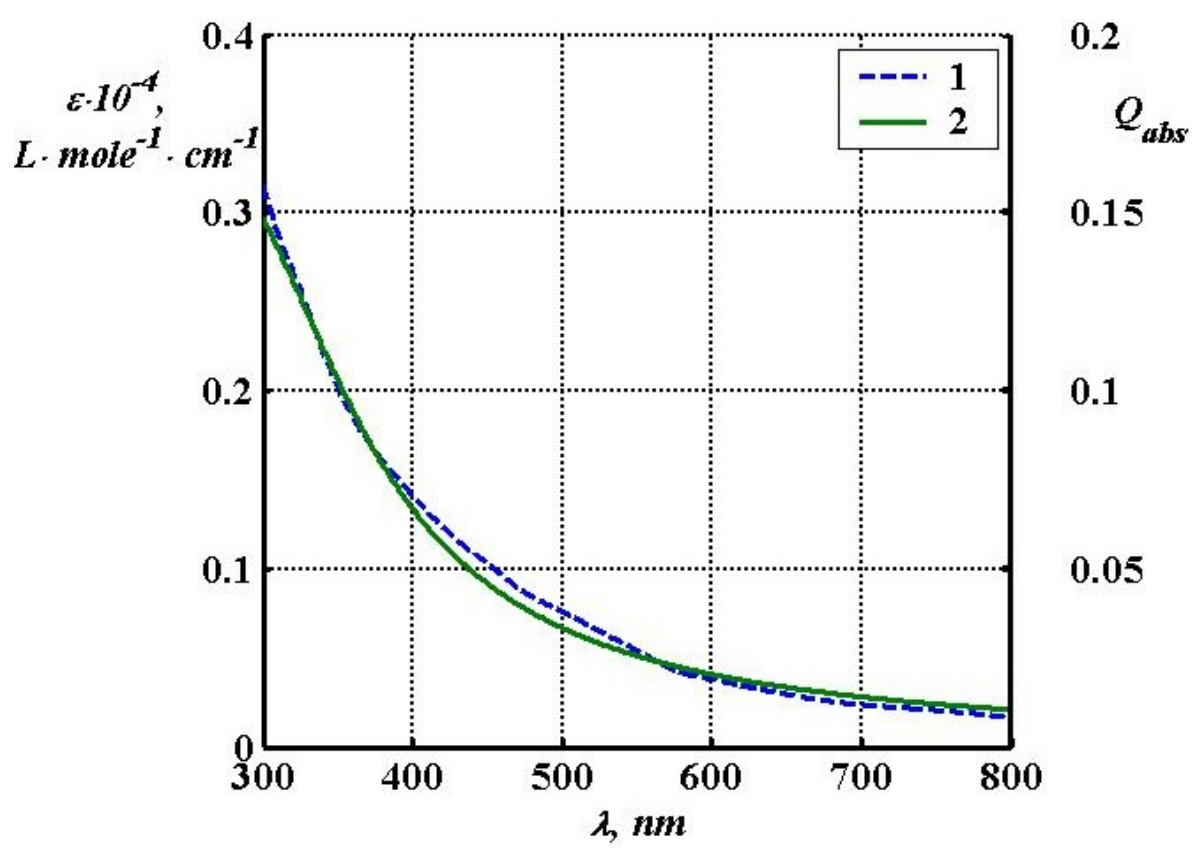

FIG. 8. Experimental spectral regularities for the molar absorptivity of $2-4$ $\mathrm{nm}$ radii cobalt nanoparticles (1) [23]; calculated spectral regularities of the absorption factor for $1.5 \mathrm{~nm}$ radii cobalt nanoparticles (2)

the increase coincides, but the quantitative results do not coincide because of the three reasons. First, cobalt is partially lost during the synthesis (yield is always less than $100 \%$ ), this makes the calculated molar absorptivity larger than that which is observed. Second, cobalt nanoparticles are covered by a mixed oxide-hydroxide film [13]. Third, the existing size distribution of the particles can distort the spectrum. When these considerations are taken into account, the calculated values and the experimental results are in good agreement. 


\section{Conclusion}

The absorption and scattering of light by cobalt nanoparticles with variation of wavelength and radius values was studied in terms of Mie theory. The optimal nanoparticles' radii performing maximal absorption and scattering efficiency factors, which are of practical interest in different applications including light filters and optic detonators, were determined for different wavelengths. The spectral dependencies of cobalt nanoparticles' scattering and absorption efficiency factors show wide bands or continuum spectrum, thus exhibiting a vast difference relative to gold or silver nanoparticles.

\section{Acknowledgments}

This work was supported by Ministry of Education and Science of the Russian Federation (governmental project No. 2014/64), grant of the Russian President (MK-4331.2015.2) and Russian Foundation for Basic Research for the financial support (grant 14-03-31648).

\section{References}

[1] Pomogajlo A.D., Rozenberg A.S., et al. Metal Nanoparticles in Polymers. Moscow, Himija, 2000,672 p.

[2] Rey J.M., Kottman J., et al. Photothermal diffuse reflectance: a new tool for spectroscopic investigation in scattering solids. Applied Physics B, 2013, 112, P. 547-551.

[3] Kalenskii A.V., Anan'eva M.V., et al. Explosive decomposition kinetics of aluminum tetranitropentaerytrite pellets. Zhurnal Tekhnicheskoi Fiziki, 2015, 85 (3), P. 119-123.

[4] Aduev B.P., Nurmukhametov D.R., et al. Integrating Sphere study of the optical properties of aluminum nanoparticles in tetranitropentaerytrite. Zhurnal Tekhnicheskoi Fiziki, 2014, 84 (9), P. 126-131.

[5] Sarkhosh L., Aleali H. et al. Large thermally induced nonlinear refraction of gold nanoparticles stabilized by cyclohexanone. Physica Status Solidi A, 2010, 207 (10), P. 2303-2310.

[6] Kalenskii A.V., Zvekov A.A., et al. Laser Wavelength Influence on the Critical Density of Initiation Energy of Energetic Materials. Fizika Goreniya i Vzryva, 2014, 50 (3), P. 98-104.

[7] Kalenskii A.V., Ananyeva M.V. Spectral regularities of the critical energy density of the pentaerythriol tetranitrate-aluminium nanosystems initiated by the laser pulse. Nanosystems: Physics, Chemistry, Mathematics, 2014, 5 (6), P. 803-810.

[8] Ananyeva M.V., Kalenskii A.V., et al Kinetic regularities of explosive decomposition of PETN contaning aluminium, cobalt and nickel nanoparticles. Bulletin of Kemerovo State University, 2014, 1-1 (57), P. 194200.

[9] Kalenskii A.V., Zykov I.Yu., et al. Explosive sensitivity of petn-aluminum composites to pulsed laser radiation. Bulletin of Kemerovo State University, 2014, 3-3 (59), P. 211-217.

[10] Aduev B.P., Nurmukhametov D.R. et al. Explosive decomposition of PETN with nanoaluminum additives under the influence of pulsed laser radiation at different wavelengths. Russian Journal of Physical Chemistry $B, 2013,7$ (4), P. 453-456.

[11] Ananyeva M.V., Zvekov A.A., et al. Promising compounds for the cap of optical detonator. Perspektivnye materialy, 2014, 7, P. 5-12.

[12] Kalenskii A.V., Kriger V.G., et al. The Microcenter Heat Explosion Model Modernization. Izvestia Vuzov Fizika, 2012, 55 (11/3), P. 62-65.

[13] Zaharov, Yu.A., Pugachev, V.M., et al. Chemical synthesis, structure and magnetic properties of nanocrystalline Fe-Co alloys. Materials Letters, 2012, 74, P. 173-175.

[14] Zvekov A.A., Kalenskii A.V., et al. Radiance distribution simulation in a transparent medium with fresnel boundaries containing aluminum nanoparticles. Komp'juternaja optika, 2014, 38 (4), P. 749-756.

[15] Zvekov A.A., Ananyeva M.V., et al. Regularities of light diffusion in the composite material pentaery thriol tetranitrate-nickel. Nanosystems: Physics, Chemistry, Mathematics, 2014, 5 (5), P. 685-691.

[16] Lukatova S.G. Calculation of the PETN-gold composites' absorptivity for the second harmonic of the ND:YAG laser. Sovremennye fundamental'nye i prikladnye issledovanija, 2014, 1 (12), P. 95-98.

[17] Kriger, V.G., Kalenskii A.V., et al. Effect of Laser Radiation Absorption E?ciency on the Heating Temperature of Inclusions in Transparent Media. Combustion, Explosion, and Shock Waves, 2012, 48 (6), P. $705-708$. 
[18] Gazenaur N.V., Zykov I.Yu., et al. The wavelength dependence of copper absorption. Aspirant, 2014, 5, P. 89-94.

[19] Kalenskii A.V., Ananyeva M.V. Absorptivity of the cobalt nanoparticles in a transparent media. Spravochnik. Inzhenernyj zhurnal s prilozheniem, 2015, 5, P. 56-60.

[20] Zolotarev V.M., Morozov V.N., et al. Optical Constants of Natural and Technical Media. 1984, Leningrad: Himija, 216 p.

[21] Nikitin A.P. Calculations of chemical parameters of thermal explosion iniciation for samples with copper nanoparticles for different wave lengths. Sovremennye fundamental'nye i prikladnye issledovanija, 2013, 4 (11), P. 68-75.

[22] Odincova O.V. Calculation of laser sensitivity of the pentaerythriol tetranitrate-silver composites. Sovremennye fundamental'nye i prikladnye issledovanija, 2014, 4 (15), P. 38-43.

[23] Ershov B.G. Metal nanoparticles in water solutions: electronic, optical and catalytic properties. Rossijskij himicheskij zhurnal, 2001, 45 (3), P. 20-30. 